\title{
Comparison between Single and Dual Femoral Fixation for Anterior Cruciate Ligament Reconstruction with a Hamstring Autograft
}

\author{
Jin Goo Kim, $\mathrm{MD}, \mathrm{PhD}^{1}$, Yong Seuk Lee, $\mathrm{MD}, \mathrm{PhD}^{2}$ and $\mathrm{Nam}$ Ki Kim, $\mathrm{MD}^{2}$ \\ Department of Orthopedic Surgery, ${ }^{1}$ Seoul Paik Hospital, Inje University College of Medicine, Seoul; ${ }^{2}$ Gil Hospital, Gachon University School of Medicine, Incheon, Korea
}

Purpose: The purpose of this study was to evaluate dual fixation with different fixation mechanisms, determine the advantages, and compare the outcomes between single and dual femoral fixation.

Materials and Methods: From April 2004 to September 2007, 19 patients who received single femoral fixation and 22 patients who received dual femoral fixation were enrolled in the study. Single femoral fixation was performed using a cross-pin expansion mechanism. Dual femoral fixation was performed using a cross-pin expansion mechanism and an EndoButton CL loop suspensory mechanism.

Results: No significant differences were found in the incidence of cross-pin problems between the two groups ( $\mathrm{p}=0.35-0.83$ ) or in the stability assessment using a KT-2000 arthrometer and pivot shift test preoperatively ( $\mathrm{p}=0.79$ and 0.77 , respectively) or postoperatively ( $\mathrm{p}=0.89$ and 0.75 , respectively). In addition, no significant differences were detected between the two groups in the Lysholm, Tegner activity, or International Knee Documentation Committee knee scores preoperatively ( $\mathrm{p}=0.07-0.47$ ) or postoperatively $(\mathrm{p}=0.15-0.89)$.

Conclusions: This study showed that dual fixation with different fixation mechanisms was not advantageous over the single fixation mechanism. Outcomes using the dual femoral fixation mechanism were not superior to those using the single femoral fixation mechanism.

Key words: Anterior cruciate ligament, Femur, Fixation, Mechanism, Comparison.

\section{Introduction}

Various options can be applied for femoral fixation of an anterior cruciate ligament (ACL) reconstruction with a hamstring autograft ${ }^{1-5}$, but each fixation mechanism has both advantages and disadvantages ${ }^{6-8)}$. The cross-pin system utilizes the expansion mechanism and is widely used ${ }^{8)}$. The pins, however, are inserted into the space between longitudinal collagen fibers, which

Received March 29, 2011; Revised (1st) April 14, 2011; (2nd) April 18, 2011; (3rd) April 24, 2011; Accepted April 28, 2011.

Correspondence to: Yong Seuk Lee, MD.

Department of Orthopedic Surgery, Gil Hospital, Gachon University School of Medicine, 117 Yong-dong, Jung-gu, Incheon 400-713, Korea. Tel: +82-32-770-1219, Fax: +82-32-764-9990

Email: smcos1@hanmail.net

This is an Open Access article distributed under the terms of the Creative Common Attribution Non-Commercial License (http://creativecommons.org/licenses/by-nc/3.0/) which permits unrestricted non-commercial use, distribution, and reproduction in any medium, provided the original work is properly cited.

Copyright $\odot$ 2011. THE KOREAN KNEE SOCIETY

www.jksrr.org can potentially allow slippage, although the significance of the slippage has not been well established ${ }^{7}$. The mechanical strength of the cross pin is comparable to other devices in experimental model $^{1,9,10)}$, but several cyclic loading tests ${ }^{1,8,11,12)}$, clinical series ${ }^{13)}$, and cases ${ }^{14,15)}$ have reported negative aspects of the cross pin. As the cortical suspensory fixation ${ }^{8}$, the EndoButton CL (Smith \& Nephew, Andover, MA, USA) is placed outside of the cortex and can be used together with the cross $\operatorname{pin}^{8)}$. Moreover, the EndoButton CL can be used as a single fixation device for femoral fixation during ACL reconstruction ${ }^{16)}$; however, it can cause tunnel widening because the joint cavity is away from the fixed area ${ }^{17-20)}$.

We have experienced some problems with the cross-pin system such as breakage and wrong posterior pin directions on followup magnetic resonance images (MRIs), and stronger fixation was needed. Dual fixation using simultaneous cross pins with the EndoButton CL was introduced to overcome these problems in the hope that each fixation would compensate for the aforementioned disadvantages.

The purpose of this study was to evaluate dual fixations with different fixation mechanisms, determine the advantages, and compare the outcomes between single and dual femoral fixation. 


\section{Materials and Methods}

\section{Demographics and Evaluation Methods}

From April 2004 to September 2007, 202 patients received hamstring autograft ACL reconstruction with cross-pin single femoral fixation or dual femoral fixation using a cross pin and EndoButton CL. Among them, we included 41 patients who had a follow-up MRI and who had no surgically managed ligament, cartilage, or meniscal injury (except for a partial meniscectomy).

We divided the patients into two groups according to the femoral fixation method (single versus dual femoral fixation groups). Single femoral fixation was performed using a cross-pin (RigidFix system $^{\circledR}$, DePuy Mitek/Johnson \& Johnson, Norwood, MA, USA) expansion mechanism (Fig. 1). Dual femoral fixation was carried out using a cross-pin expansion mechanism and the EndoButton CL closed loop (Smith \& Nephew, Andover, USA) suspensory mechanism (Fig. 2).

We compared the follow-up MRI between the two groups based on the problems (>1 mm slippage on the coronal plane, breakage of the pin on the coronal plane, and a posteriorly directed cross pin to the posterior condylar line on an axial image). MRI results were compared to evaluate whether dual fixation was helpful for overcoming the disadvantages of the cross pin. Preoperative and final follow-up clinical and stability results were evaluated between the two groups. Stability results were evaluated with a KT-2000 arthrometer (MEDmetric, San Diego, CA, USA) and a pivot-shift test. Clinical results were evaluated with the Lysholm, International Knee Documentation Committee (IKDC) subjective score and Tegner activity score.

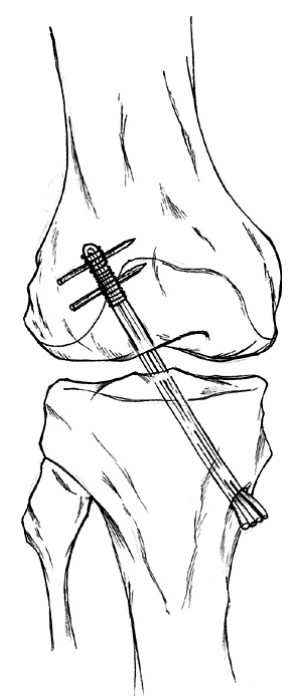

Fig. 1. The cross-pin expansion mechanism was only used in the single femoral fixation group.

\section{Surgical Procedure for Dual Femoral Fixation and \\ Rehabilitation}

All patients underwent arthroscopic surgery with spinal or general anesthesia. After a thorough evaluation of the cartilage and meniscus status, proper surgical procedures were performed if necessary. Semitendinosus and gracilis hamstring autografts were harvested and prepared with a quadruple strand coupled with the EndoButton CL. Four free ends were sutured with Ethibond no. 2 sutures using an interlocking whip stitch technique, and the folded end was sutured with Vicryl no. 2 (VICRYL, Ethicon, Somerville, NJ, USA) sutures after coupling it with the EndoButton CL. After removing the remnant medial wall ACL tissue from the lateral femoral condyle, we marked an entry point for the femoral tunnel using a microfracture awl at the 10 to $10: 30$ oclock position on the right knee and 1:30 to 2 oclock position on the left knee. A tibial tunnel was created using an ACL drill guide positioned just anterior to the medial collateral ligament to make an oblique femoral tunnel. We started reaming the tibial tunnel using a $2 \mathrm{~mm}$ smaller than the graft diameter, and the tunnel was dilated up to the anticipated diameter using a serial tunnel dilator in increments of $0.5 \mathrm{~mm}$ to achieve a press-fit fixation. The final tunnel length for the graft was determined after measuring the total length of the femoral tunnel. Most of the graft tunnels of femoral and tibial sides were 8-8.5 mm diameter and 30-35 mm length. After drilling both the tibial and femoral tunnels, the hamstring graft was passed from a distal to proximal direction. When the EndoButton CL was retrieved out of the femoral cortex and confirmed by toggling the EndoButton CL, the graft was firmly pulled out in the distal

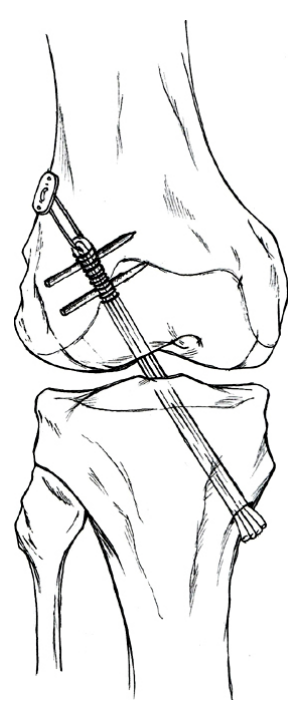

Fig. 2. The cross pin and EndoButton CL were used concomitantly in the dual fixation group. 
direction so that it was placed tightly on the femoral cortex. Two rigid-fix pins were inserted with firm tension on the graft. After performing pretensioning by pulling the graft distally and cycling the knee 15 times, the distal end was fixed into the tibial tunnel with an interference bioabsorbable screw and a metal staple. Tibial fixation was done using a bioabsorbable screw and it was usually $1 \mathrm{~mm}$ larger in diameter because the tibial tunnel tended to be dilated slightly while making the bone tunnels. All patients immediately began active quadriceps isometric exercises and active range of motion exercises. The patients were allowed tolerable weight bearing with full extension locked in a brace for 3 weeks after the surgery. Crutch walking was maintained for 6 weeks. At 3 weeks, $90^{\circ}$ motion was allowed and at 6 weeks, $120^{\circ}$ motion was allowed. At 3 months, straight line running was allowed and at 6 months, we recommended that the patients participate in sports activity.

\section{Statistical Methods}

A difference of more than $3 \mathrm{~mm}$ on the KT-2000 arthrometer or 10 points on the IKDC score was clinically meaningful. The alpha was 0.05 and the power was 0.8 . The required sample size was 21 cases. The analysis was performed as a nonparametric test using the SPSS ver. 12.0 (SPSS Inc., Chicago, IL, USA). The chi-square test was used to compare MRI problems, and the t-test was used to compare stability and the clinical results. A p-value $<0.05$ was considered significant.

Table 1. Fixational Problems in Each Group

\begin{tabular}{lcccccc}
\hline & \multicolumn{2}{c}{ Single fixation $(\mathrm{n}=19)$} & & \multicolumn{2}{c}{ Dual fixation $(\mathrm{n}=22)$} \\
\cline { 2 - 3 } \cline { 5 - 6 } & Positive & Negative & & Positive & Negative \\
\hline Slippage $(>1 \mathrm{~mm})$ & 1 & 18 & & 4 & 18 \\
Breakage & 6 & 13 & & 8 & 14 \\
Posterior direction & 4 & 15 & & 6 & 16 \\
Total problem & 8 & 11 & & 10 & 12 \\
\hline
\end{tabular}

\section{Results}

Fifteen men and 4 women were in the single fixation group; their mean age at the time of the operation was 32.7 years (range, 17 to 51 years). The mean follow-up was 28 months (range, 18 to 52 months). Eighteen men and 4 women were in the dual fixation group; their mean age at the time of the operation was 29.3 years (range, 18 to 41 years). The mean follow-up period was 34 months (range, 22 to 60 months).

Detailed comparisons of the fixational problems between the two groups are listed in Table 1. Some patients had more than one problem (32\%). No significant differences were observed in the incidence of slippage $(\mathrm{p}=0.35)$, breakage $(\mathrm{p}=0.75)$, posterior direction $(\mathrm{p}=0.73)$, or total problems $(\mathrm{p}=0.83)$.

Preoperatively, no significant differences existed between the two groups in the assessment of the Lysholm score $(\mathrm{p}=0.47)$, IKDC subjective score $(\mathrm{p}=0.07)$, Tegner activity score $(\mathrm{p}=0.28)$, KT 2000 arthrometer evaluation ( $\mathrm{p}=0.79$ ), or the pivot-shift test ( $\mathrm{p}=0.77$ ). Postoperatively, no significant differences were detected between the two groups in the assessment of the Lysholm score $(\mathrm{p}=0.59)$, IKDC subjective score $(\mathrm{p}=0.15)$, Tegner activity score $(\mathrm{p}=0.18)$, KT 2000 arthrometer evaluation $(\mathrm{p}=0.89)$, or the pivotshift test $(\mathrm{p}=0.75)$ (Table 2$)$.

\section{Discussion}

The principal finding of this study was that dual fixation with different fixation mechanisms was neither advantageous nor helpful for overcoming the disadvantages of single fixation with cross pins. Anatomic aperture fixation with an interference screw at the original ACL insertion site has rationale due to the disadvantages of extra-articular indirect graft fixation. In some hamstring graft fixation studies, interference screws have provided sufficient initial fixation strength during biomechanical tests $^{12,21-23)}$, but a possible disadvantage of this fixation method is the contact of the screws with the intra-articular cavity.

Table 2. Outcome Analysis between the Single and Dual Fixation Groups

\begin{tabular}{|c|c|c|c|c|}
\hline & \multicolumn{2}{|c|}{ Singe fixation $(n=19)$} & \multicolumn{2}{|c|}{ Dual fixation $(\mathrm{n}=22)$} \\
\hline & Pre-operation & Last follow-up & Pre-operation & Last follow-up \\
\hline KT 2000 & 3.837 & 1.51 & 4.459 & 1.91 \\
\hline Pivot grade & 1.63 & 0.26 & 1.83 & 0.36 \\
\hline Lysholm & 67.68 & 90.68 & 71.23 & 90.32 \\
\hline Tegner & 2.74 & 5.47 & 1.95 & 4.63 \\
\hline IKDC & 56.77 & 87.36 & 53.32 & 74.21 \\
\hline
\end{tabular}

IKDC: International Knee Documentation Committee. 
Some studies have reported inflammatory reactions after using this fixation method ${ }^{12,24)}$. In the cross-pin method, the grafts are fixed by two biodegradable pins piercing tendon strands perpendicularly ${ }^{12)}$. The biomechanical properties of the cross pin are acceptable and superior to the interference screw for femoral fixation of the hamstring graft ${ }^{7,8,10,11)}$ with good clinical results $^{2}$. However, negative aspects of cross pins have also been reported. The maximal failure load is inferior to the suspensory mechanism and the failure mechanism is pin fracture ${ }^{1,3,8,12)}$. Choi et al. ${ }^{13)}$ reported that pin breakage is a common phenomenon, and broken cross pins are a factor related to the laxity of ACL reconstruction. Some case reports have reported pin breakage and loose intra-articular migration of pins ${ }^{14,15)}$. We also experienced some problems in follow-up MRIs such as breakage and posteriorly directed pins even though they showed good clinical results and stability. Therefore, we concluded that stronger fixation would be needed in active persons and that these must be compared to clinical and stability results.

The EndoButton CL is included in the cortical suspensory fixation $^{8)}$. This fixation mechanism has some merits for overcoming disadvantages of the expansion mechanism. The EndoButton CL has comparable mechanical strength but can cause tunnel widening due to a $2-3 \mathrm{~mm}$ graft-tendon micromotion under a physiological range load in which the articular surface is away from the fixed area, although many possible causes of tunnel widening exist ${ }^{17-20)}$.

Therefore, we used the dual fixation method to overcome the disadvantages. Our comparison is reliable because we used the same rehabilitation protocol in the two groups, so that only the fixation method was different. No significant difference was found between the two groups, contrary to our expectations, and dual fixation had no additional advantage. This suggested that the graft-tunnel fixation was fairly stable and contributed to early healing due to the use of press-fit fixation with a tunnel dilator in $0.5 \mathrm{~mm}$ increments during surgery. Using the single fixation with cross pins may have provided sufficient stability because we only allowed full extension with a locked brace for 3 weeks and partial weight bearing with crutches for 6 weeks without applying an accelerated rehabilitation protocol postoperatively. The most notable finding in our study was that although breakage occurred, the posterior directed pin position did not result in clinical instability during single fixation, implying that the initial press-fit fixation and proper protection are more critical than additional fixation mechanism. Our results do not apply to accelerated rehabilitation, and the fixation differences under this condition are beyond the scope of this study.
However, our study had several limitations: the sample size was relatively small according to the power analysis and the study was limited to patients who checked a MRI. Therefore, some selection and randomization bias could not be eliminated.

\section{Conclusions}

This study showed that dual fixation with different fixation mechanisms was neither advantageous for overcoming the disadvantages of the single fixation mechanism nor superior in terms of the outcomes.

\section{References}

1. Ahmad CS, Gardner TR, Groh M, Arnouk J, Levine WN. Mechanical properties of soft tissue femoral fixation devices for anterior cruciate ligament reconstruction. Am J Sports Med. 2004;32:635-40.

2. Ahn JH, Park JS, Lee YS, Cho YJ. Femoral bioabsorbable cross-pin fixation in anterior cruciate ligament reconstruction. Arthroscopy. 2007;23:1093-9.

3. Becker R, Voigt D, Starke C, Heymann M, Wilson GA, Nebelung W. Biomechanical properties of quadruple tendon and patellar tendon femoral fixation techniques. Knee Surg Sports Traumatol Arthrosc. 2001;9:337-42.

4. Fabbriciani C, Milano G, Mulas PD, Ziranu F, Severini G. Anterior cruciate ligament reconstruction with doubled semitendinosus and gracilis tendon graft in rugby players. Knee Surg Sports Traumatol Arthrosc. 2005;13:2-7.

5. Fabbriciani C, Mulas PD, Ziranu F, Deriu L, Zarelli D, Milano G. Mechanical analysis of fixation methods for anterior cruciate ligament reconstruction with hamstring tendon graft. An experimental study in sheep knees. Knee. 2005;12:135-8.

6. Lee YS, Ahn JH, Kim JG, Park JH, Park JW, Kim CB, Lee SW. Analysis and prevention of intra-operative complications of TransFix fixation in anterior cruciate ligament reconstruction. Knee Surg Sports Traumatol Arthrosc. 2008;16:639-44.

7. Kim JG, Lee YS, Kim YJ, Shim JC, Ha JK, Park HA, Yang SJ, Oh SJ. Correlation between the rotational degree of the dial test and arthroscopic and physical findings in posterolateral rotatory instability. Knee Surg Sports Traumatol Arthrosc. 2010:18:123-9.

8. Milano G, Mulas PD, Ziranu F, Piras S, Manunta A, Fabbriciani C. Comparison between different femoral 
fixation devices for ACL reconstruction with doubled hamstring tendon graft: a biomechanical analysis. Arthroscopy. 2006;22:660-8.

9. Dargel J, Schmidt-Wiethoff R, Heck M, Bruggemann GP, Koebke J. Comparison of initial fixation properties of sutured and nonsutured soft tissue anterior cruciate ligament grafts with femoral cross-pin fixation. Arthroscopy. 2008;24:96-105.

10. Zantop T, Weimann A, Wolle K, Musahl V, Langer M, Petersen W. Initial and 6 weeks postoperative structural properties of soft tissue anterior cruciate ligament reconstructions with cross-pin or interference screw fixation: an in vivo study in sheep. Arthroscopy. 2007;23:14-20.

11. Kousa P, Jarvinen TL, Vihavainen M, Kannus P, Jarvinen M. The fixation strength of six hamstring tendon graft fixation devices in anterior cruciate ligament reconstruction. Part I: femoral site. Am J Sports Med. 2003;31:174-81.

12. Zantop T, Weimann A, Rummler M, Hassenpflug J, Petersen W. Initial fixation strength of two bioabsorbable pins for the fixation of hamstring grafts compared to interference screw fixation: single cycle and cyclic loading. Am J Sports Med. 2004;32:641-9.

13. Choi NH, Lee JH, Victoroff BN. Do broken cross-pins compromise stability after anterior cruciate ligament reconstructions with hamstring tendons? Arthroscopy. 2007;23:1334-40.e2.

14. Han I, Kim YH, Yoo JH, Seong SC, Kim TK. Broken bioabsorbable femoral cross-pin after anterior cruciate ligament reconstruction with hamstring tendon graft: a case report. Am J Sports Med. 2005;33:1742-5.

15. Cossey AJ, Paterson RS. Loose intra-articular body following anterior cruciate ligament reconstruction. Arthroscopy. 2005;21:348-50.

16. Williams RJ 3rd, Hyman J, Petrigliano F, Rozental T, Wickiewicz TL. Anterior cruciate ligament reconstruction with a four-strand hamstring tendon autograft. J Bone Joint
Surg Am. 2004;86:225-32.

17. Barrett GR, Papendick L, Miller C. Endobutton button endoscopic fixation technique in anterior cruciate ligament reconstruction. Arthroscopy. 1995;11:340-3.

18. L'Insalata JC, Klatt B, Fu FH, Harner CD. Tunnel expansion following anterior cruciate ligament reconstruction: a comparison of hamstring and patellar tendon autografts. Knee Surg Sports Traumatol Arthrosc. 1997;5:234-8.

19. Muneta T, Sekiya I, Yagishita K, Ogiuchi T, Yamamoto H, Shinomiya K. Two-bundle reconstruction of the anterior cruciate ligament using semitendinosus tendon with endobuttons: operative technique and preliminary results. Arthroscopy. 1999;15:618-24.

20. Webster KE, Feller JA, Hameister KA. Bone tunnel enlargement following anterior cruciate ligament reconstruction: a randomised comparison of hamstring and patellar tendon grafts with 2-year follow-up. Knee Surg Sports Traumatol Arthrosc. 2001;9:86-91.

21. Brand J Jr, Weiler A, Caborn DN, Brown CH Jr, Johnson DL. Graft fixation in cruciate ligament reconstruction. Am J Sports Med. 2000;28:761-74.

22. Brown GA, Pena F, Grontvedt T, Labadie D, Engebretsen L. Fixation strength of interference screw fixation in bovine, young human, and elderly human cadaver knees: influence of insertion torque, tunnel-bone block gap, and interference. Knee Surg Sports Traumatol Arthrosc. 1996;3:238-44.

23. Hoher J, Scheffler SU, Withrow JD, Livesay GA, Debski RE, Fu FH, Woo SL. Mechanical behavior of two hamstring graft constructs for reconstruction of the anterior cruciate ligament. J Orthop Res. 2000;18:456-61.

24. Weiler A, Windhagen HJ, Raschke MJ, Laumeyer A, Hoffmann RF. Biodegradable interference screw fixation exhibits pull-out force and stiffness similar to titanium screws. Am J Sports Med. 1998;26:119-26. 